\title{
Cerebral Hernia Caused By Epidural Hygroma Due to Excessive Drainage of Cerebrospinal Fluid: A Rare Case and Literature Review
}

Qiang-ping Wang ${ }^{1}$, Jun-peng $\mathrm{Ma}^{2}$, Jun-wen Guan ${ }^{2}$ and Chao You

${ }^{1}$ Department of Neurosurgery, Union Hospital, Tongji Medical College, Huazhong, University of Science and Technology, 1277 JieFang Avenue, Wuhan 430022, P.R. China ${ }^{2}$ Department of Neurosurgery, West China Hospital, Sichuan University, Chengdu 610041, P.R. China

\begin{abstract}
The cerebral hernia is usually caused by intracranial hematoma, tumor, abscess. Here, we report a rare case of cerebral hernia caused by severe epidural hygroma. The patient was a 51-year-old male, with a large, right-sided skull defect and severe scalp depression, who had received a decompressive craniectomy for traumatic brain injury and a lumboperitoneal shunt for secondary hydrocephalus 6 months earlier. A cranioplasty with titanium mesh was performed after admitted. Unfortunately, he suffered from a complication of epidural fluid collection. Through repeated local treatment of the hygroma, the patient was not better but rather became more and more serious, and developed to cerebral hernia eventually. However, after finding out the root cause, the patient got dramatically improvement just by elevating the valve pressure of the lumboperitoneal shunt. The case and literature review are instructive to the treatment of patients with skull bone defect associated with hydrocephalus.
\end{abstract}

Keywords: Cerebral hernia; Epidural hygroma; CSF drainage; Cranioplasty

\section{Introduction}

Decompressive craniectomies have been done more frequently because of their known benefits in lowering intracranial pressure directly and quickly [1]. However, some patients may develop communicating hydrocephalus and require cerebrospinal fluid (CSF) shunting treatment including ventriculo-peritoneal (V-P) shunt and lumbar drainage [2]. After this procedure, the bulging craniectomy area may become extremely depressed, which may result in complications such as intracranial hematoma and subdural effusion when doing a cranioplasty subsequently $[3,4]$. We report a rare case of serious, stubborn epidural hygroma secondary to a cranioplasty in a patient with skull bone defect and CSF diversion device. And we also reviewed literature, summarizing the methods to prevent and treat this complication, to acquaint neurosurgeons with this situation.

\section{Case Report}

A 51-year-old male patient was admitted with a large, right-sided skull defect and severe scalp depression (Figure 1A). He suffered from a traumatic brain injury 6 month earlier and undergone decompressive craniectomy. Hydrocephalus developed 4 weeks after the operation and he received a lumboperitoneal shunt subsequently. A cranioplasty with titanium mesh was performed. Unfortunately, the postoperative computed tomographic (CT) scan revealed that he got a complication of epidural fluid collection (Figure 1B). After 3 days of observation, recheck of CT found that the epidural fluid collection increased. A percutaneous needle aspiration was performed and $30 \mathrm{ml}$ light red fluid was pumped out. However, CT scan showed no change of the epidural hygroma 3 days later. So, we adopted a 3 -day continuous percutaneous drainage and about $20 \mathrm{ml}$ fluid was drained out every day. Nevertheless, the patient was deteriorated not to contact eye, obey command and unresponsive to noxious stimuli, and his right pupil became dilated on the third day after drainage termination. The emergency CT scan revealed marked increase of collection and marked herniation to the left (Figure 1C). After an emergency discussion, a conclusion of excessive drainage of CSF was produced. And a percutaneous needle aspiration and continuous drainage were performed and the opening pressure of lumboperitoneal shunt was elevated from 120 to 140 $\mathrm{mm} \mathrm{H}_{2} \mathrm{O}$ immediately. Within 1 hour, his condition improved. $\mathrm{He}$

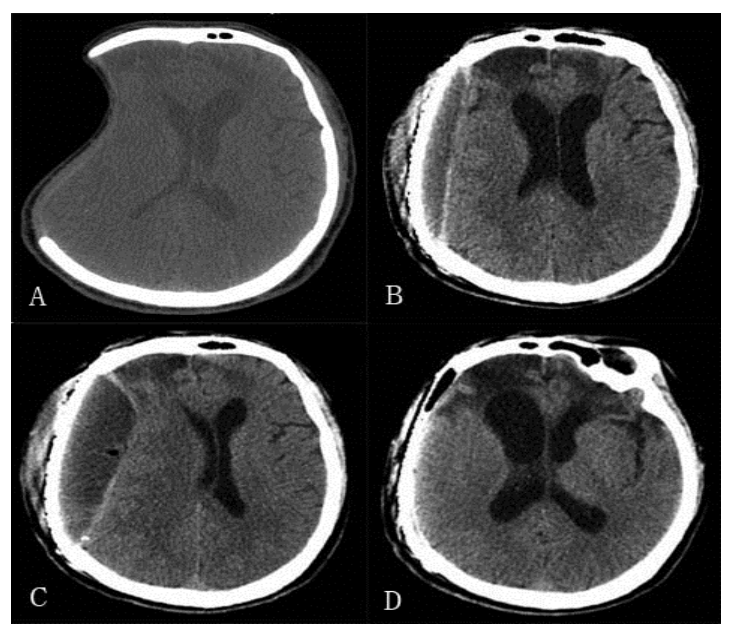

Figure 1: (A) Thin section axial CT scan images obtained before cranioplasty. (B) Axial CT scans showing epidural CSF collection secondary to cranioplasty. (C) Repeat brain CT scan showing deterioration of epidural CSF collection with midline shift. (D) Follow-up brain CT scan a week afterward showing absolutely absorption of the epidural collection.

became alert and follow-up brain CT scan a week afterward confirmed absolutely absorption of the epidural hygroma (Figure 1D). After 6 months of follow-up, the patient was well and the valve pressure of the lumboperitoneal shunt stayed the same.

\section{Discussion}

The cerebral hernia is usually caused by intractable intracranial

${ }^{*}$ Corresponding author: Chao You, Department of Neurosurgery, West China Hospital, Sichuan University, Chengdu 610041, P.R. China, Tel: +86159-28063038; Fax: +86028-8712-1111; E-mail: kouxiangtang7595@163.com

Received June 15, 2017; Accepted July 12, 2017; Published July 15, 2017

Citation: Wang Q, Ma J, Guan J, You C (2017) Cerebral Hernia Caused By Epidural Hygroma Due to Excessive Drainage of Cerebrospinal Fluid: A Rare Case and Literature Review. J Neurol Disord 5: 353. doi:10.4172/2329-6895.1000353

Copyright: @ 2017 Wang Q, et al. This is an open-access article distributed under the terms of the Creative Commons Attribution License, which permits unrestricted use, distribution, and reproduction in any medium, provided the original author and source are credited. 
hypertension in patients suffering from serious head injury, stroke, and cerebral swelling due to vasospasm after a subarachnoid haemorrhage and so on [1]. As far as we know, there was no report about hernia caused by hygroma. Hygroma is a complication that can occur after head trauma and secondary to cranioplasty [5]. However, it is usually located in the subdural rather than in the epidural space. For this case, the CT scan showed that epidural hygroma caused the herniation, which was rarely reported. In fact, excessive drainage of CSF played a critical role in the development of the herniation. Firstly, the severe brain tissue depression made the tight adhesion of titanium mesh and dura impossible, and the dura fistula caused by surgery leaded CSF collection in the dead space between dura and titanium mesh. Secondly, the valve pressure set was lower and intracanial hypotension occurred after the cranioplasty. Then the pressure gradient between the dead space and the intracerebral content caused by excessive drainage of CSF made the dura fistula a one-way valve. That's why the CSF was excessive drained but the epidural fluid collection became increasingly. Therefore, repeatedly percutaneous needle aspiration and continuous drainage didn't work, and the collection became more serious and caused cerebral hernia eventually. However, the patient got dramatically improvement just by stopping excessive drainage of CSF.

Decompressive craniectomies have been done more frequently for intractable intracranial hypertension in patients suffering from serious head injury, stroke, cerebral swelling due to vasospasm after a subarachnoid haemorrhage and so on [1]. However, some of these patients may develop communicating hydrocephalus. Consequently, most of them require CSF shunting treatment [2]. After this procedure, the bulging craniectomy area may become extremely depressed after shunting due to the decrease in ventricular size $[3,6]$. And it may increase the risk of complications such as intracranial hematoma and subdural effusion when doing a cranioplasty subsequently [3].

Cranioplasty is a simple operation in neurosurgery. However, many authors reported complications and morbidity after cranioplasty $[7,8]$. As stated above, cranioplasty for patients with hydrocephalus implanted with shunting device is more apt to suffer from complications. So, what can we do to prevent these occur? First, expanding the concave space at the skull defect may be an effective way to eliminate the dead space between the skull plate and the dura to facilitate the cranioplasty, which makes the tight adhesion of titanium mesh and dura compassable. Liao et al. reported 4 cases getting favourable prognosis by clipping of the shunt tube provisionally and doing operation after the expansion of the depressed scalp [6]. Second, the operation details are also crucial for the clinical outcome. Damage of the dura should be avoided during operation, and we should repair it when it happens to prevent CSF leakage. Suturing of the dura and strapping it with the titanium mesh is also an effective way to eliminate the dead space, which can reduce the risk of postoperative bleeding and fluid collection. In our case, the tight adhesion of titanium mesh and dura is impossible because we did not take any measures against the severe skull concavity, which made a huge dead space. And the dura fistula caused by surgery leaded CSF collection. Finally, when the situation occurs, we should identify the root cause of the problem and solve it, but not focus on the superficial phenomena.

\section{Conclusion}

We presented a rare case of cerebral hernia caused by severe epidural hygroma, who got recovery through appropriate control of intracranial pressure. The case illustrated the importance of maintaining proper intracranial pressure during preoperational stage of cranioplasty in patients with CSF shunting.

\section{References}

1. Fields JD, Lansberg MG Skirboll SL, Kurien PA, Wijman CA (2006) "Paradoxical" transtentorial herniation due to CSF drainage in the presence of a hemicraniectomy. Neurology 67: 1513-1514.

2. Waziri A, Fusco D, Mayer SA, McKhann GM II (2007) Postoperative hydrocephalus in patientsy udergoing decompresive hemicraniectomy for ischemic or hemorrhagic stroke. Neurosurgery 61: 489-493.

3. Liao CC, Kao MC (2002) Cranioplasty for patients with severe depressed skul bone defect after cerebrospinal fluid shunting. J Clin Neurosci 9: 553-555.

4. Tabaddor K, LaMorgese J (1976) Complications of a large cranial defect. J Neurosurg 44: 506-508.

5. Lee KS (1998) The pathogenesis and clinical significance of traumatic subdural hygroma. Brain Inj 12: 595-603.

6. Han PY, Kim JH, Kang HI, Kim JS (2008) "Syndrome of the sinking skinflap" secondary to the ventriculoperitoneal shunt after craniectomy. J Korean Neurosurg Soc 43: 51-53.

7. Chang V, Hartzfeld P, Langlois M, Mahmood A, Seyfried D (2010) Outcomes of cranial repair after craniectomy. J Neurosurg 112: 1120-1124.

8. Huh PW, Yoo DS, Cho KS, Park CK, Kang SG, et al. (2006) Diagnostic method for differentiating external hydrocephalus from simple subdural hygroma. J Neurosurg 105: 65-70. 